\title{
Digital Health in Cardiology: Time for Action
}

\author{
Lise Solberg Nes ${ }^{\mathrm{a}-\mathrm{c}}$ \\ aDivision of Medicine, Center for Shared Decision Making and Collaborative Care Research, Oslo University Hospital, \\ Oslo, Norway; ${ }^{\mathrm{b}}$ Institute of Clinical Medicine, Faculty of Medicine, University of Oslo, Oslo, Norway; ${ }^{\mathrm{c}}$ Department of \\ Psychiatry and Psychology, College of Medicine and Science, Mayo Clinic, Rochester, MN, USA
}

What is the current status of digital health in cardiology, and what kind of role should digital health have in cardiology in the future?

\section{A Call for Advancing and Implementing Digital Health in Cardiology}

In the current issue of Cardiology, Lotman and Viigimaa [1] present a brief overview of the Estonian perspective of digital health in cardiology. Digital health is a broad term, defined by the World Health Organization as "...a term encompassing eHealth" (i.e., "the use of information and communications technology in support of health and health-related fields") and mHealth (i.e., "the use of mobile wireless technologies for public health"), as well as emerging areas, such as the use of advanced computing sciences in "big data," genomics and artificial intelligence," [2; p. 1].

There is little doubt that digital health solutions can hold promise for the future of cardiovascular care. The question is how, when, and where. This is not new. In 2013, Cowie et al. [3] stated that the engagement of car- diologists all over Europe was "urgently required," and that the time for the cardiology community to engage in eHealth had arrived.

The current action plan for the European Society of Cardiology (ESC) involves playing a pro-active role in digital health in support of cardiovascular health in Europe. The ESC e-Cardiology working group has recently published a paper aiming to guide cardiologists and other stakeholders in the digital health area; it provides a brief overview of existing cardiology-related digital health applications, pointing to current challenges for application implementation, and presents ideas on how to proceed [4]. Similarly, the current Innovation Strategy of the American College of Cardiology (ACC) states that ACC should be "leading the digital transformation of healthcare" and points to the need for effective and safe innovations aiming to improve efficiency of care and improve patient-physician relations, supported by appropriate payment models [5].

While the demand for more modern and user-friendly digital healthcare services is evident, digital innovations are abundant, and the potential for digital health innovations apparently is immense, the digital transformation of healthcare delivery is still slow-moving.

\section{KARGER}

(c) 2019 S. Karger AG, Basel 


\section{Why Is the Progress of Digital Health Seemingly Dragging?}

Healthcare involves patients; people who depend on clinicians, the healthcare system, and on quality of care. This means that innovations must be rigorously tested and deemed safe and effective prior to implementation. Furthermore, healthcare is complicated, systems multifaceted, and the workload of providers demanding, aspects not readily facilitating or supporting change. Considering these factors, the availability of digital health solutions alone will not cause implementation of new technology or transformation of healthcare delivery.

\section{Need for Evidence of Effect}

The general consumer market is inundated with digital solutions, including smartphone applications, smart watches, wearables (e.g., activity/sleep trackers), and "smart" programs (e.g., blood pressure monitors and glucose sensors). Yet, the evidence of effect from digital health technology in healthcare services is still at an early stage, and clinical adoption requires evidence. In order to implement digital health solutions in cardiology, there is an urgent need for evidence, including large-scale studies, demonstrating that these new solutions can in fact improve outcomes.

\section{Barriers to Implementation of Digital Health in Cardiology}

Recent international conferences have focused on opportunities and barriers related to digital health implementation in cardiology, and the ESC e-Cardiology working group has a strong voice in this process, pointing to the endorsement by healthcare providers as crucial for digital health implementation [4].

A majority of participants in a recent national survey among members of the Netherlands Society of Cardiology considered eHealth as clinically beneficial and likely to improve patient satisfaction. However, the participating clinicians expressed concern that eHealth solutions might increase their workload, concern over lacking reimbursement models, and also expressed a need for integration of new and existing systems (e.g., medical records) [6].

Concerns about digital health solutions have also been raised by patients with cardiovascular disease. The ESC
e-Cardiology working group reflects upon these concerns as potentially relating to health status, personal motivation, low digital literacy, low education, and low socioeconomic status [4]. There are also concerns by patients related to security and privacy, quality of care, accessibility to digital tools, and about potential impersonal aspects of digital healthcare [4].

Supporting the stance of the ESC e-Cardiology working group, a recent realist systematic review of implementation strategies in eHealth programs for patients with chronic illness pointed to management support and engagement, as well as training of clinicians, internal and external facilitation (i.e., engagement, problem solving, support), and providing/soliciting audit and feedback, as important for implementation success [7].

\section{Digital Solutions for Clinical Settings}

Despite the abundance of digital health solutions in the general consumer market, few of these solutions are designed or tested for clinical settings or developed with stakeholder (i.e., patient/provider) involvement. Even though the effect of digital health technologies on cardiovascular outcomes is still undetermined, research has shown digital solutions to support self-management for people living with other health conditions $[8,9]$, and the potential for self-management of cardiovascular risk factors (e.g., physical activity) is vast.

A recent scoping review identified behavior change techniques and persuasive system design principles used in eHealth interventions, as well as techniques and principles used to stimulate motivation, behavior change, and adherence in support of weight loss/maintenance [10]. eHealth interventions with significant effects used selfmonitoring, feedback, goal setting, and shaping knowledge in combination with social support in support of healthy behaviors [10], aspects of importance when considering self-management for patients with cardiovascular disease as well.

Considering the heavy workload of most clinicians, there is a strong need for new digital solutions to be integrated into existing clinical solutions $[4,6]$. There have also been suggestions for utilization of digital health system to aid in decision support, as well as e-consultations to enable cooperation and support between primary care physicians and specialists. In addition, there is the potential of artificial intelligence, and intelligence displayed by machines may play a major role in the future of cardiovascular imaging and potential individual monitoring devices [11]. 


\section{User-Centered Digital Health Focus}

Digital health solutions in cardiology must be developed focusing on the end users (i.e., patients and healthcare providers). While a number of digital health interventions and solutions aiming to support patients with various types of health challenges exist, the involvement of potential end users in the design and development phases is still rare. This means that developers cannot be sure to meet the needs of actual end users.

Employing a user-centered design during the development of digital solutions may involve interviewing potential end users about their experiences, needs, and preferences, as well as potential facilitators and barriers for using such solutions [12]. Digital health delivery also presents with challenges regarding health literacy and/or digital literacy. Addressing the intersection between electronic health and digital health literacy, an 18-point "Digital Universal Precautions" has been presented and suggested as a "mandate for healthcare organizations" committed to supporting digital health literacy [13].

\section{Reimbursement Incentives}

The rise in life expectancy is associated with rising healthcare costs. Digital healthcare solutions could potentially ease this challenge through options such as video consultations, offsite monitoring, and digital self-management programs. The lack of reimbursement models for digital healthcare services is a major barrier to the implementation of new services, however, and reimbursement methods/regulations must be in place for digital healthcare services to substitute/support parts of healthcare in the future.

\section{Safety and Data Protection Regulations}

While the options provided by digital healthcare are plentiful and exciting, digital health introduces a range of data protection issues. Even countries with high levels of e-governance and cybersecurity face challenges with the protection of patient privacy, and the potential impact of cyber threats is high for digitally dependent countries.

The General Data Protection Regulation (GDPR) regulates data protection and privacy for citizens of the European Union (EU), also the transfer of personal data to areas outside the EU. The overall aim of the GDPR is to give individuals control over own personal data and to provide international business with overarching regulations within the EU. Other regulations apply in other regions of the world. For example, there is no equivalent to the GDPR in the United States, but a variety of different state and federal rules govern some of the same issues.

The tendency for impatience and frustration by patients, healthcare providers, researchers, and businesses towards safety, privacy, and data protection regulations can be understandable. However, for digital healthcare technologies to reach full potential, solutions must meet strict safety, privacy, and data protection regulations.

\section{The Road Ahead for Digital Health in Cardiology}

In summary, there is a need for evidence that digital health technologies are in fact effective and can improve outcomes in cardiology. There is also a need for digital solutions for clinical settings, that is, digital solutions that are designed to address clinical problems and can be integrated into clinical care. These developments should at least in part be driven by user (i.e., patients and healthcare providers) centeredness. There is also a need for incentive for use, including reimbursement models for digital healthcare services. Finally, there is a serious need for attention to data protection regulations, including privacy and security.

\section{Disclosure Statement}

The author has no potential conflicts of interest to disclose.

\section{Funding Sources}

The study has not received any funding.

References

1 Lotman E-M, Viigimaa M. Digital Health in Cardiology: The Estonian Perspective. Cardiology. DOI: 1011.59/000504564.

2 WHO guideline: recommendations on digital interventions for health system strengthening. Geneva: World Health Organization; 2019. Licence: CC BY-NC-SA 3.0 IGO.

3 Cowie MR, Chronaki CE, Vardas P. e-Health innovation: time for engagement with the cardiology community. Eur Heart J. 2013 Jul; 34(25):1864-8.

4 Frederix I, Caiani EG, Dendale P, Anker S, Bax J, Böhm A, et al. ESC e-Cardiology Working Group Position Paper: overcoming challenges in digital health implementation in cardiovascular medicine. Eur J Prev Cardiol. 2019 Jul;26(11):1166-77. 
5 Walsh MN, Rumsfeld JS. Leading the digital transformation of healthcare: the ACC innovation strategy. J Am Coll Cardiol. 2017 Nov; 70(21):2719-22.

6 Treskes RW, Wildbergh TX, Schalij MJ, Scherptong RW. Expectations and perceived barriers to widespread implementation of e-Health in cardiology practice: results from a national survey in the Netherlands. Neth Heart J. 2019 Jan;27(1):18-23.

7 Varsi C, Solberg Nes L, Kristjansdottir OB, Kelders SM, Stenberg U, Zangi HA, et al. Implementation strategies to enhance the implementation of eHealth programs for patients with chronic illnesses: realist systematic review. J Med Internet Res. 2019 Sep;21(9): e14255.
8 Børøsund E, Varsi C, Clark MM, Ehlers SE, Adrykowski MA, Sætre Sleveland HR, et al. Pilot testing an app-based stress management intervention for cancer survivors. Transl Behav Med. 2019 Apr;bz062.

9 Vugts MA, Joosen MC, van der Geer JE, Zedlitz AM, Vrijhoef HJ. The effectiveness of various computer-based interventions for patients with chronic pain or functional somatic syndromes: A systematic review and metaanalysis. PLoS One.2018 May;13(5):e0196467.

10 Asbjørnsen RA, Smedsrød ML, Solberg Nes L, Wentzel J, Varsi C, Hjelmesæth J, et al. Persuasive system design principles and behavior change techniques to stimulate motivation and adherence in electronic health interventions to support weight loss maintenance: Scoping review. J Med Internet Res. 2019 Jun; 21(6):e14265.
11 Schoenhagen P, Mehta N. Big data, smart computer systems, and doctor-patient relationship. Eur Heart J. 2017 Feb;38(7):508-10.

12 Ledel Solem IK, Varsi C, Eide H, Kristjansdottir OB, Mirkovic J, Børøsund E, et al. Patients' needs and requirements for eHealth pain management interventions: Qualitative study. J Med Internet Res. 2019 Apr; 21(4):e13205.

13 Smith B, Magnani JW. New technologies, new disparities: the intersection of electronic health and digital health literacy. Int J Cardiol. 2019 Oct;292:280-2. 